\title{
TUNABLE HYBRID OPTICAL MODES IN A BOUNDED CHOLESTERIC LIQUID CRYSTAL WITH THE TWIST DEFECT
}

\author{
Maxim V. Pyatnov ${ }^{1,2}$, Stepan Ya. Vetrov ${ }^{2,3}$, and Ivan V. Timofeev ${ }^{1,3}$ \\ ${ }^{1}$ Institute of Nanotechnology, Spectroscopy and Quantum Chemistry, Siberian Federal \\ University, 660041 Krasnoyarsk, Russia \\ ${ }^{2}$ Institute of Engineering Physics and Radio Electronics, Siberian Federal University, 660041 \\ Krasnoyarsk, Russia \\ ${ }^{3}$ Kirensky Institute of Physics, Federal Research Center KSC SB RAS, 660036 Krasnoyarsk, Russia \\ e-mail: MaksPyatnov@yandex.ru \\ Coupling between the defect mode of a cholesteric liquid crystal and the localized mode of the \\ cholesteric liquid crystal-phase plate-metal structure is theoretically demonstrated. It is shown that \\ the transmittance spectrum can be tuned by changing the twist defect angle and helix pitch, which \\ are governed by external factors. The spectra for different circular polarizations of the incident light \\ are different; specifically, at the nondiffracting polarization, there is no defect-mode transmittance \\ peak.
}

\section{INTRODUCTION}

In recent years, the interactions between different optical resonances have been intensively investigated. One of such resonances is often the optical Tamm state called also the Tamm plasmon. It is implemented when the light is locked at the interface between a photonic crystal and a medium with the negative permittivity [1, 2] or between two photonic band gap materials [3]. Such localized modes at the interface between two highly-reflecting materials can be formed by both TE and TM linear polarizations and excited at any light incidence angles. In experiments, a Tamm plasmon can be observed as a narrow resonance in the transmittance or reflectance spectrum of a sample. The keen interest in Tamm plasmons is due to their high potential for application in lasing [4] and sensing [5].

Different variants of coupling of Tamm plasmons with other modes, e.g., cavity modes, exciton polaritons [6-8], and surface plasmons $[9,10]$, were proposed. These hybrid modes are characterized by the anticrossing of resonances upon tuning the position of one of them. The position of a Tamm plasmon can be tuned by changing the thickness of a top layer adjacent to the metal or the thickness of the 
metallic film. The tunable hybrid Tamm microcavities were studied by Pankin et al. [11]. Liu et al. demonstrated coupling between the localized surface plasmons with hybrid Tamm plasmons [12]. Zhang et al. proposed to use the hybrid Tamm plasmon polariton/microcavity modes in white top-emitting organic light-emitting devices [13]. Mischok et al. demonstrated a novel near-infrared detector design based on a Tamm plasmon polariton microcavity to optically enhance the charge-transfer state absorption in the bulk heterojunction of an organic photodiode [14]. Kumar et al. proposed a coupled Tamm-plasmon-polariton (TPP) hybrid-mode-based selfreferenced refractive-index sensor [15].

The sensitivity of liquid crystals to the external factors makes them promising for application in optical systems. Cholesteric liquid crystals (CLCs), similar to onedimensional photonic crystals, have a photonic band gap [16, 17]. At the normal incidence of light, the band gap is the only for the light with the diffracting circular polarization coinciding with the cholesteric helix twist. The radiation with the nondiffracting circular polarization is not reflected from such a structure. The band gap exists in the wavelength range of $p n_{o}<\lambda<p n_{e}$, where $p$ is the helix pitch, $n_{o}$ and $n_{e}$ are the ordinary and extraordinary CLC refractive indices, and $\lambda$ is the optical wavelength in a medium. The helix pitch is the distance after which the director pointing the preferred orientation of molecules turns by $2 \pi$.

The defects embedded in the ideal CLC structure lead to the occurrence of narrow transmission bands in the band gap, which correspond to the localized defect modes. There are several ways of inducing the photonic defect modes in a CLC. This can be obtained by using a thin layer of an isotropic [18-20] or anisotropic substance [21-23] placed between two CLC layers, a twist defect (jump of the angle of rotation of the cholesteric helix) [24-27], or a defect caused by the local CLC helix pitch change [28-30]. The photonic defect modes in a CLC containing a combination of several defects were studied in [31-33].

The transmission bands corresponding to the localized defect modes occur at the circular polarization of light with the twist consistent with the CLC helix twist. At the opposite circular polarization, the spectrum contains deeps at the frequencies of the transmission peaks. 
The advantage of a CLC is its tunability. The CLC structure can be changed by the temperature variation, external electric and magnetic fields, or photo-orientation effect. In particular, one can change the cholesteric helix pitch and, thus, tune the band gap position. In addition, the defect variation can be used to tune the spectral properties of a structure.

Previously, some attempts were made to obtain a Tamm plasmon at the interface between a CLC and a metallic layer. We demonstrated the possibility of existence of the localized optical state (optical Tamm state) in the CLC-quarter-wave phase plate-metallic film system [34, 35], hereinafter referred to as CLCPPMeS. The need in a phase plate is dictated by the polarization features of light reflection from the CLC and metal. The light reflected from the CLC preserves its circular polarization, while the transmission through the metal changes it. In this system, the maximum field localization is observed at the phase plate/metal interface. The role of a phase plate can be played by the additional oppositely-handed CLC layer [36] or a planar anisotropic defect in the CLC [37, 38].

In this work, we investigate coupling of the CLC defect modes with the CLCPPMeS localized modes and formation of tunable hybrid localized optical modes. The defect mode is induced by the twist defect of a crystal structure.

\section{DESCRIPTION OF THE MODEL}

The structure under study is shown in Fig. 1. It consists of a right-handed CLC with the structural twist defect, a quarter-wave phase plate, and a metallic film. The CLC parameters used in the simulation involved extraordinary and ordinary refractive indices of $n_{e}=1.71$ and $n_{o}=1.54$, respectively, and a helix pitch of $p=0.4$ $\mu \mathrm{m}$. The parameters correspond to the mixture of a chiral center (Merck, S-811) and a nematic liquid crystal (Merck, E44) [39]. The band-gap center of the specified CLC is positioned at a wavelength of $\lambda=650 \mathrm{~nm}$. The CLC length is $4 \mu \mathrm{m}$ and there is a twist defect (helical phase jump) at the layer center. The pitch numbers on the left and on the right from the defect are the same. The twist defect size is specified by angle $\alpha$. The CLC layer was assumed to rotate clockwise at the observation along the light propagation direction. 
Let the CLC refractive indices $n_{e}$ and $n_{o}$ be equal to the corresponding indices of the phase plate. The quarter-wave plate thickness is $d=\lambda_{0} / 4\left(n_{e}-n_{o}\right)=0.96 \mu \mathrm{m}, \lambda_{0}-$ длина волны в вакууме. The structure is surrounded by a medium with the refractive index equal to the averaged CLC refractive index $n_{e x t}=\left(n_{e}+n_{o}\right) / 2$. The metallic film thickness is $d_{\mathrm{m}}=50 \mathrm{~nm}$ and the film permittivity is specified by the Drude approximation

$$
\varepsilon(\omega)=\varepsilon_{0}-\frac{\omega_{p}^{2}}{\omega(\omega+i \gamma)},
$$

where $\varepsilon_{0}=5$ is the ionic core contribution, $\hbar \omega_{\mathrm{p}}=9 \mathrm{eV}$ is the plasma frequency, and $\gamma=$ $0.02{ } \mathrm{~V}$ is the reciprocal relaxation time [40]. These parameters are typical of silver.

The simplest way of obtaining the proposed structure to use polymer cholesteric elastomers [41] instead of conventional CLCs. Schmidtke et al. [27] and Castro-Garay et al. [42] experimentally observed the photonic defect modes induced by the twist structural defect in such cholesteric materials. The twist defect can be formed by rotation of one part of the elastomer relative to the other part around the helix axis so that the director variation continuity is broken. The spectral properties of such materials, similar to the properties of conventional CLCs, can be changed by using various factors $[43,44]$.

The combination of the quarter-wave plate and metallic film can be replaced by a polarization-preserving mirror, as was proposed in [45].

\section{RESULTS AND DISCUSSION}

The transmittance spectra and field intensity distribution for the structure were determined using the transfer matrix method for calculating stratified anisotropic structures proposed by Berreman [46].

Figure $2 \mathrm{a}$ presents the calculated transmittance spectrum recorded upon variation in the twist-defect angle $\alpha$ for the right-handed circular polarization of incident light. In the calculation, the first CLC layer was rotated, while the second layer adjacent to the phase plate was fixed. In the absence of a twist defect $(\alpha=0)$, the spectrum only contains a peak at a wavelength of $643 \mathrm{~nm}$, which corresponds to the 
CLCPPMeS localized mode. As the $\alpha$ value increases, the defect-mode peak shifts to the long-wavelength region. At $\alpha=1.38 \mathrm{rad}$, the wavelengths of the two peaks coincide and the resonance frequency splits. The split peaks are positioned at wavelengths of 637 and $649 \mathrm{~nm}$ (inset in Fig. 2a). Upon variation in the $\alpha$ value, the spectrum demonstrates the anticrossing typical of hybrid states.

The white dashed line in the plot shows the position of the resonance wavelength for the CLC with a CLC twist defect [32]

$$
\lambda=\frac{\lambda_{2}+\lambda_{1}}{2}+\frac{\lambda_{2}-\lambda_{1}}{2} \cos \alpha
$$

where $\lambda_{2}$ and $\lambda_{1}$ are the long- and short-wavelength boundaries of the CLC band gap. Since the investigated CLC layer is merely $4-\mu \mathrm{m}$-thick, its band gap is broader than $p n_{o}<\lambda<p n_{e}$ and, consequently, we have $\lambda_{2}-\lambda_{1} \neq p\left(n_{e}-n_{o}\right)$. This fact was used when plotting the white dashed line in Fig. 2a.

At the incidence of light with the left-handed circular polarization, the different spectrum is obtained (Fig. 2b). The transmittance peak corresponding to the CLC defect mode is absent. This is due to the fact that, as we mentioned above, the spectrum of the light with the nondiffracting circular polarization contains a weak deep. Therefore, when the frequencies of the peaks corresponding to the CLCPPMeS localized mode and CLC defect mode coincide, the splitting does not occur.

Figure 3 shows the spatial distribution of the squared absolute value of electric field $|\mathrm{E}|^{2}(\mathrm{z})$ for a wavelength of $649 \mathrm{~nm}$ at $\alpha=1.38 \mathrm{rad}$ for both circular polarizations of the incident light. In both cases, the field is localized on the twist defect and at the plate/metal interface. However, at the incidence of light with the right-handed circular polarization, the field is localized much stronger. At $637 \mathrm{~nm}$, the $|E|^{2}(z)$ distribution is similar.

Changing the helix pitch by using external factors, one can effectively tune the CLC transmittance spectrum. We calculated the transmittance spectrum for the structure shown in Fig. 1 at different CLC helix pitches (Fig. 4). As was shown in [34], the condition for the existence of localized optical modes in the CLCPPMeS is 


$$
\frac{2 \pi\left(n_{e}-n_{o}\right) d}{\lambda}=\frac{\pi}{2} .
$$

If the parameters of the CLC and phase plate are strongly different and this ratio is not valid, there are no optical states localized at the phase plate/metal interface. Therefore, at the helix pitches significantly different from $400 \mathrm{~nm}$, the spectrum only contains the peak corresponding to the CLC defect mode.

The change in the phase plate thickness leads to the change in the transmittance spectrum of the structure. We studied the properties of the structure containing a phase plate with a thickness of $d^{\prime}=5 d=4.8 \mu \mathrm{m}$. The plate remains quarter-wave relative to a wavelength of $\lambda_{0}=650 \mathrm{~nm}$ and a birefringence of 0.17 . At this plate thickness, the spectrum includes several peaks corresponding to the localized modes of the CLC-phase plate-metal system. Changing the twist defect, one can tune the transmittance spectrum of the investigated structure (Fig. 5).

\section{CONCLUSION}

Thus, we demonstrated the existence of hybrid optical modes formed by the CLC defect mode and localized modes of the CLC-phase plate-metal structure. The defect mode is excited by the twist defect of the cholesteric structure. We showed the possibility of tuning the transmittance spectrum structure CLCPPMeS by changing the twist-defect angle and CLC helix pitch. The helix pitch can be changed using external factors, e.g., electric field. The spectra for the light with different circular polarizations are different, because the resonance corresponding to the defect mode at the nondiffracting circular polarization of light has a deep instead of a transmittance peak.

\section{ACKNOWLEDGMENTS}

The reported study was funded by Russian Foundation for Basic Research, Government of Krasnoyarsk Territory, Krasnoyarsk Region Science and Technology Support Fund to the research project № 17-42-240464. 
1. M. Kaliteevski, I. Iorsh, S. Brand, R. A. Abram, J. M. Chamberlain, A. V. Kavokin, and I. A. Shelykh, Tamm plasmon-polaritons: possible electro-magnetic states at the interface of a metal and a dielectric Bragg mirror, Phys. Rev. B 76, 165415 (2007).

2. I. V. Timofeev, P. S. Pankin, S. Y. Vetrov, V. G. Arkhipkin, W. Lee, and V. Y. Zyryanov, Chiral Optical Tamm States: Temporal Coupled-Mode Theory, Crystals 7, 113 (2017).

3. T. Goto, A. V. Dorofeenko, A. M. Merzlikin, A. V. Baryshev, A. P. Vinogradov, M. Inoue, A. A. Lisyansky, and A. B. Granovsky, Optical Tamm states in one-dimensional magnetophotonic structures, Phys. Rev. Lett. 101,113902 (2008).

4. A. R. Gubaydullin, C. Symonds, J. Bellessa, K. A. Ivanov, E. D. Kolykhalova, M. E. Sasin, A. Lemaitre, P. Senellart, G. Pozina, and M. A. Kaliteevski, Enhancement of spontaneous emission in Tamm plasmon structures, Sci. Rep. 7, 9014 (2017).

5. S.-G. Huang, K.-Ping Chen, and S.-C. Jeng, Phase sensitive sensor on Tamm plasmon devices, Opt. Mater. Exp. 7, 1267 (2017).

6. M. Kaliteevski, S. Brand, R. A. Abram, I. Iorsh, A. V. Kavokin, and I. A. Shelykh, Hybrid states of Tamm plasmons and exciton polaritons, Appl. Phys. Lett. 95, 251108 (2009).

7. M. Wurdack, N. Lundt, M. Klaas, V. Baumann, A. V. Kavokin, S. Höfling, and C. Schneider, Observation of hybrid Tamm-plasmon exciton-polaritons with GaAs quantum wells and a $\mathrm{MoSe}_{2}$ monolayer, Nat. comm. 8, 259 (2017).

8. Sk. S.-U. Rahman, T. Klein, S. Klembt, J. Gutowski, D. Hommel, and K. Sebald, Observation of a hybrid state of Tamm plasmons and microcavity exciton polaritons, Sci. Rep. 6, 34392 (2016).

9. B. I. Afinogenov, V. O. Bessonov, A. A. Nikulin, and A. A. Fedyanin, Observation of hybrid state of Tamm and surface plasmon-polaritons in one-dimensional photonic crystals, Appl. Phys. Lett. 103, 061112 (2013).

10. S. Azzini, G. Lheureux, C. Symonds, J.-M.Benoit, P. Senellart, A. Lemaitre, J.-J. Greffet, C. Blanchard, C. Sauvan, and J. Bellessa, Generation and spatial control of hybrid Tamm plasmon/surface plasmon modes, ACS Photonics 3, 1776 (2016).

11. H. Liu, J. Gao, Z. Liu, X. Wang, H. Yang, and H. Chen, Large electromagnetic field enhancement achieved through coupling localized surface plasmons to hybrid Tamm plasmons, J. Opt. Soc. Am. B 32, 2061 (2015).

12. P. S. Pankin, S. Ya. Vetrov, and I. V. Timofeev, Tunable hybrid Tamm-microcavity states. J. Opt. Soc. Am. B 34, 2633 (2017).

13. X. L. Zhang, J. Feng, X. C. Han, Y. F. Liu, Q. D. Chen, J. F. Song, and H. B. Sun, Hybrid Tamm plasmon-polariton/microcavity modes for white top-emitting organic light-emitting devices, Optica 2, 579 (2015).

14. A. Mischok, B. Siegmund, D. S. Ghosh, J. Benduhn, D. Spoltore, M. Böhm, H.Fröb, C. Körner, K. Leo, and K. Vandewal, Controlling Tamm Plasmons for Organic Narrowband NearInfrared Photodetectors, ACS Photonics 4, 2228 (2017).

15. S .Kumar, M. K. Shukla, P. S.Maji, and R. Das, Self-referenced refractive index sensing with hybrid-Tamm-plasmon-polariton modes in sub-wavelength analyte layers, J. Phys. D: Appl. Phys. 50, 375106 (2017). 
16. V. A. Belyakov, Diffraction Optics of Complex Structured Periodic Media, (Springer Verlag, New York, 1992).

17. L. M. Blinov, Structure and Properties of Liquid Crystals, Chap. 12 (Springer, 2011).

18. Y. C. Yang, C. S. Kee, J. E. Kim, H. Y. Park, J. C. Lee, and Y. J. Jeon, Photonic defect modes of cholesteric liquid crystals, Phys. Rev. E 60, 6852 (1999).

19. S. M. Jeong, N. Y. Ha, Y. Takanishi, K. Ishikawa, H. Takezoe, S. Nishimura, and G. Suzaki, Defect mode lasing from a double-layered dye-doped polymeric cholesteric liquid crystal films with a thin rubbed defect layer, Appl. Phys. Lett. 90, 261108 (2007).

20. V. A. Belyakov and S. V. Semenov, Optical defect modes in chiral liquid crystals, J. Exp. Theor. Phys. 112, 694 (2011).

21. A. Lakhtakia, V. C. Venugopal, and M. W. McCall, Spectral holes in Bragg reflection from chiral sculptured thin films: Circular polarization filters, Opt. Commun. 177, 57 (2000).

22. A. H. Gevorgyan, and M. Z. Harutyunyan, Chiral photonic crystals with an anisotropic defect layer, Phys. Rev. E 76, 031701 (2007).

23. M. H. Song, B. Park, K. C. Shin, T. Ohta, Y. Tsunoda, H. Hoshi, Y. Takanishi, K. Ishikawa, J. Watanabe, S. Nishimura, T. Toyooka, Z. Zhu, T.M. Swager, and H. Takezoe, Effect of Phase Retardation on Defect-Mode Lasing in Polymeric Cholesteric Liquid Crystals, Adv. Mat. 16, 779 (2004).

24. V. I. Kopp, and A. Z. Genack, Twist defect in chiral photonic structures, Phys. Rev. Lett. 89, 033901 (2002).

25. I. J. Hodgkinson, Q. H. Wu, K. E. Thorn, A. Lakhtakia, and M. W. McCall, Spacerless circularpolarization spectral-hole filters using chiral sculptured thin films: theory and experiment, Opt. Comm. 184, 57 (2000).

26. M. Becchi, S. Ponti, J. A. Reyes, and C. Oldano, Defect modes in helical photonic crystals: An analytic approach, Phys. Rev. B 70, 033103 (2004).

27. J. Schmidtke, W. Stille, and H. Finkelmann, Defect mode emission of a dye doped cholesteric polymer network, Phys. Rev. Lett. 90, 083902 (2003).

28. A. V. Shabanov, S. Ya. Vetrov, and A. Yu. Karneev, Reflection spectrum of a cholesteric liquid crystal with structural defects, JETP Lett. 80, 181 (2004).

29. T. Matsui, M. Ozaki, and K. Yoshino, Tunable photonic defect modes in a cholesteric liquid crystal induced by optical deformation of helix, Phys. Rev. E 69, 061715 (2004).

30. Y. C. Hsiao, H. T. Wang, and W. Lee, Thermodielectric generation of defect modes in a photonic liquid crystal, Opt. Exp. 22, 3593 (2014).

31. I. J. Hodkinson, Q. H. Wu, L. De Silva, and A. Matthew, Supermodes of Chiral Photonic Filters with Combined Twist and Layer Defects, Phys. Rev. Lett. 91, 223903 (2003).

32. J. Schmidtke and W. Stille, Photonic defect modes in cholesteric liquid crystal films, Eur. Phys. J. E 12, 553 (2003).

33. S. Ya. Vetrov, M. V. Pyatnov, and I. V. Timofeev, Photonic defect modes in a cholesteric liquid crystal with a resonant nanocomposite layer and a twist defect. Phys. Rev. E 90, 032505 (2014).

34. S. Ya. Vetrov, M. V. Pyatnov, and I. V. Timofeev, Surface modes in "photonic cholesteric liquid crystal-phase plate-metal” structure, Opt. Lett. 39, 2743 (2014). 
35. S. Ya. Vetrov, M. V. Pyatnov, and I. V. Timofeev, Spectral and polarization properties of a 'cholesteric liquid crystal—phase plate—metal'structure, J. Opt. 18, 015103 (2015).

36. M. V. Pyatnov, S. Ya. Vetrov, and I. V. Timofeev, Localised optical states in a structure formed by two oppositely handed cholesteric liquid crystal layers and a metal, Liq. Cryst. 44, 674 (2017).

37. M. V. Pyatnov, S. Ya. Vetrov, and I. V. Timofeev, Localized optical modes in a defectcontaining liquid-crystal structure adjacent to the metal. J. Opt. Soc. Am. B 34, 2011 (2017).

38. M. V. Pyatnov, S. Ya. Vetrov, and I. V. Timofeev, Localized optical states in a liquid-crystal structure adjacent to a metal, Opt. and Spectr. 123, 189 (2017).

39. Y. Matsuhisa, R. Ozaki, K. Yoshino, and M. Ozaki, High Q defect mode and laser action in one-dimensional hybrid photonic crystal containing cholesteric liquid crystal, Appl. Phys. Lett, 89, 101109 (2006).

40. P. B. Johnson and R. W. Christy, Optical constants of the noble metals, Phys. Rev. B 6, 4370 (1972).

41. M. Warner and E. M. Terentjev, Liquid Crystal Elastomers, Chap. 9. (Clarendon, Oxford, 2003).

42. P. Castro-Garay, J. A. Reyes, and A. Corella-Madueño, Twist defect in an imprinted cholesteric elastomer. Appl. Phys. Lett. 94, 163504 (2009).

43. A. M. Menzel, and H. R. Brand, Cholesteric elastomers in external mechanical and electric fields, Phys. Rev. E 75, 011707 (2007).

44. H. Nagai, and K. Urayama, Thermal response of cholesteric liquid crystal elastomers. Phys. Rev. E 92, 022501 (2015).

45. I. V. Timofeev, and S. Ya. Vetrov, Chiral optical Tamm states at the boundary of the medium with helical symmetry of the dielectric tensor, JETP Lett. 104, 380 (2016).

46. D. W. Berreman, Optics in Stratified and Anisotropic Media: 4 X 4-Matrix Formulation, J. Opt. Soc. Am. 62, 502 (1972). 


\section{FIGURES}

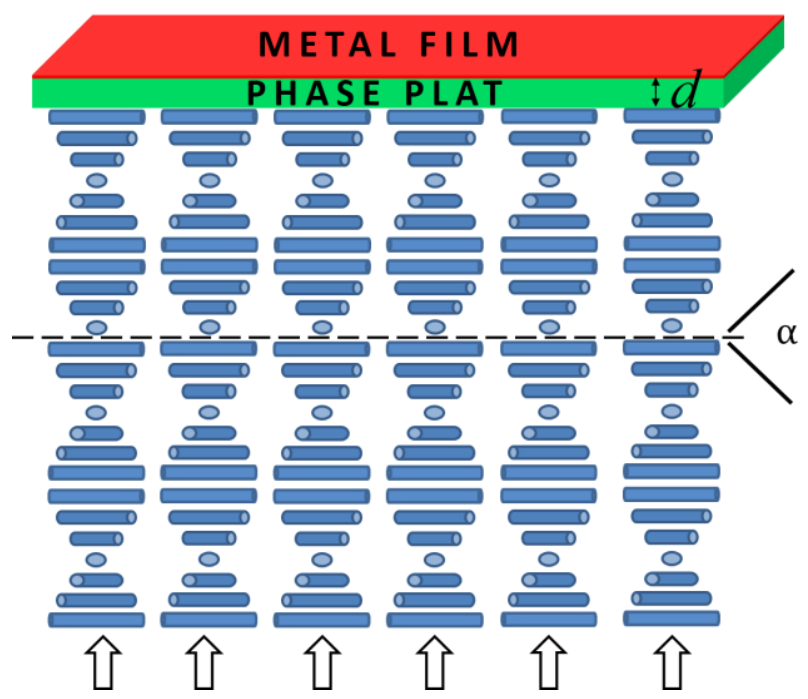

Fig. 1. Schematic of the structure.
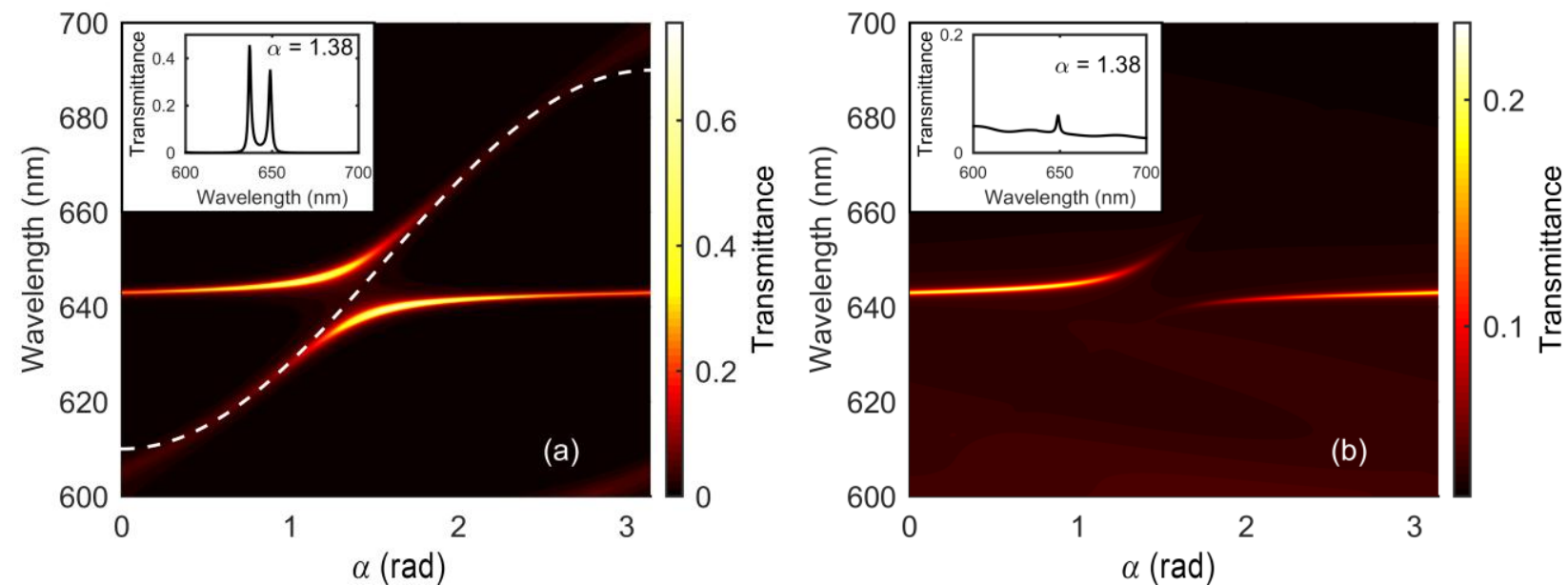

Fig. 2. Transmittance of the structure (Fig.1) as a function of twist defect angle $\alpha$ for (a) the rightand (b) left-handed circular polarizations of incidence light. The first CLC layer is rotated and the CLC layer adjacent to the phase late is fixed. Inset: transmittance spectrum at $\alpha=1.38 \mathrm{rad}$; the resonance wavelengths correspond to the CLCPPMeS localized mode and CLC defect mode.

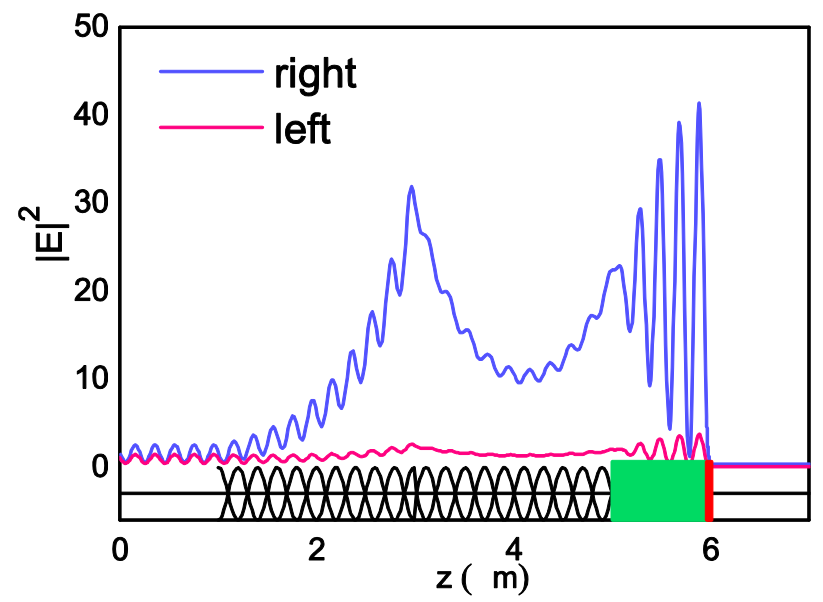

Fig. 3. Spatial local electric field intensity distribution in the structure from Fig.1 for the right- and left-handed circular polarizations of incidence light at a wavelength of $649 \mathrm{~nm}$. 


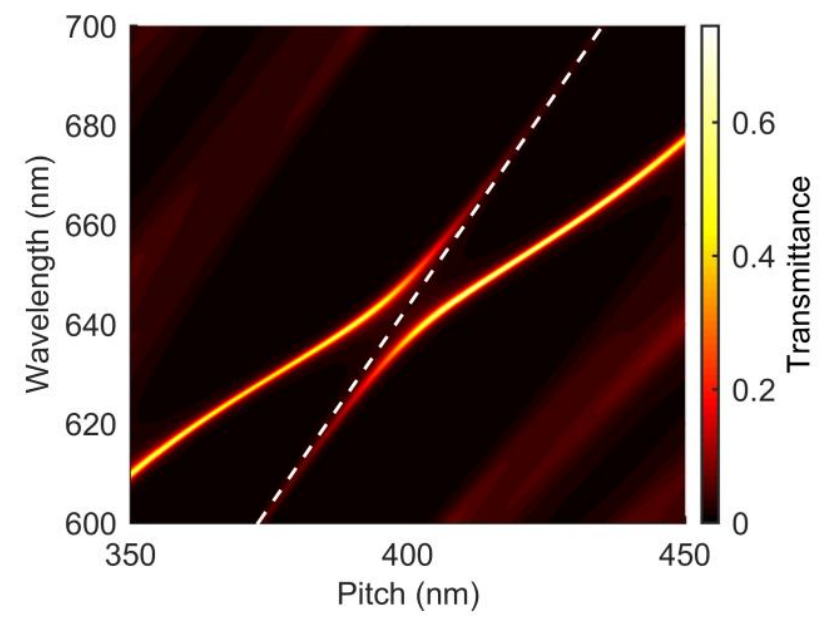

Fig. 4. Transmittance of the structure from Fig. 1 as a function of the CLC helix pitch. The white dashed line shows the position of the CLC defect mode peak calculated using formula (2) at $\alpha=1.38 \mathrm{rad}$.

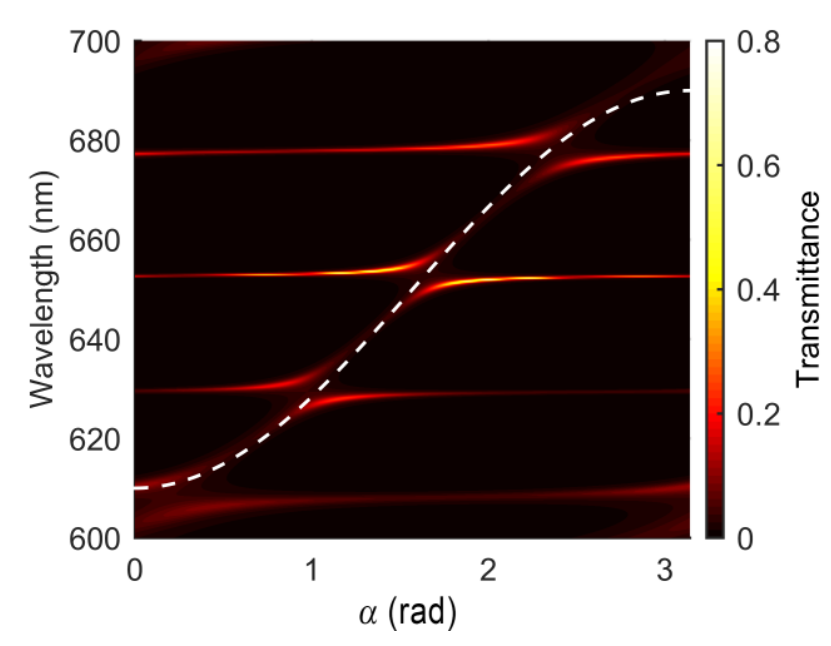

Fig. 5. Transmittance of the structure from Fig. 1 as a function of the twist-defect angle for the right-handed circular polarization of incidence light. The first CLC layer is rotated and the CLC layer adjacent to the phase plate is fixed. The white dashed line shows the position of the CLC defect mode peak calculated using formula (2). $d^{\prime}=5 d$. 


\section{FIGURES CAPTIONS}

Fig. 1. Schematic of the structure.

Fig. 2. Transmittance of the structure (Fig.1) as a function of twist defect angle $\alpha$ for (a) the right- and (b) left-handed circular polarizations of incidence light. The first CLC layer is rotated and the CLC layer adjacent to the phase late is fixed. Inset: transmittance spectrum at $\alpha=1.38 \mathrm{rad}$; the resonance wavelengths correspond to the CLCPPMeS localized mode and CLC defect mode.

Fig. 3. Spatial local electric field intensity distribution in the structure from Fig.1 for the right- and left-handed circular polarizations of incidence light at a wavelength of $649 \mathrm{~nm}$.

Fig. 4. Transmittance of the structure from Fig. 1 as a function of the CLC helix pitch. The white dashed line shows the position of the CLC defect mode peak calculated using formula (2) at $\alpha=1.38 \mathrm{rad}$.

Fig. 5. Transmittance of the structure from Fig. 1 as a function of the twist-defect angle for the right-handed circular polarization of incidence light. The first CLC layer is rotated and the CLC layer adjacent to the phase plate is fixed. The white dashed line shows the position of the CLC defect mode peak calculated using formula (2). $d^{\prime}=5 d$. 